\title{
A New Numerical Method to Solve Nonlinear Volterra-Fredholm Integro-Differential Equations
}

\author{
Jinjiao $\mathrm{Hou}^{a}$, Jing $\mathrm{Niu}^{a}$, Minqiang $\mathrm{Xu}^{b}$ and \\ Welreach Ngolo ${ }^{a}$ \\ ${ }^{a}$ School of Mathematics and Sciences, Harbin Normal University \\ 150025 Harbin, China \\ ${ }^{b}$ College of Science, Zhejiang University of Technology \\ 310014 Hangzhou, China \\ E-mail: h27950288@163.com \\ E-mail(corresp.): njirwin@163.com \\ E-mail: mqxu math@126.com \\ E-mail: welreachngolo@gmail.com
}

Received June 10, 2020; revised June 9, 2021; accepted June 9, 2021

\begin{abstract}
In this paper, a new method combining the simplified reproducing kernel method (SRKM) and the homotopy perturbation method (HPM) to solve the nonlinear Volterra-Fredholm integro-differential equations (V-FIDE) is proposed. Firstly the HPM can convert nonlinear problems into linear problems. After that we use the SRKM to solve the linear problems. Secondly, we prove the uniform convergence of the approximate solution. Finally, some numerical calculations are proposed to verify the effectiveness of the approach.
\end{abstract}

Keywords: nonlinear Volterra-Fredholm integro-differential equations, simplified reproducing kernel method, homotopy perturbation method.

AMS Subject Classification: 45G10; 45J05.

\section{Introduction}

This article mainly discusses the nonlinear V-FIDE:

$$
Y u(x)+H(u(x))=y(x), \quad u(a)=\alpha,
$$

Copyright (C) 2021 The Author(s). Published by Vilnius Gediminas Technical University

This is an Open Access article distributed under the terms of the Creative Commons Attribution License (http://creativecommons.org/licenses/by/4.0/), which permits unrestricted use, distribution, and reproduction in any medium, provided the original author and source are credited. 
where

$$
\begin{gathered}
Y u(x)=u^{\prime}(x)+q(x) u(x), \\
H(u(x))=\lambda_{1} \int_{0}^{x} K_{1}(x, t) F(u(t)) d t+\lambda_{2} \int_{0}^{1} K_{2}(x, t) G(u(t)) d t .
\end{gathered}
$$

The parameters $\lambda_{1}, \lambda_{2}$ are constants. $F(u(x))$ and $G(u(x))$ are constant coefficient polynomials of $u(x)$. The V-FIDE has been widely used in physics, biological and engineering $[1,2,3,10,16]$. In order to obtain accurate numerical solutions more quickly, many methods for solving such problems have been proposed in recent years. Maleknejad [10] introduced the hybrid functions method. Babolian [2,3] proposed the triangular functions method and the operational matrix with block-pulse functions. Hybrid Legendre polynomials and block-pulse functions approach were used by Maleknejad [9]. Bakodah [4] discussed the Laplace discrete Adomian decomposition method over the integro-differential equation. Biazar and Ghanbari [5] presented He's homotopy perturbation method. Bildik [6] used the modified decomposition method to obtain the approximate solution of nonlinear V-FIDE. Ghasemi [8] formulated homotopy perturbation method for solving nonlinear equations. In recent years, with the development of reproducing kernel space theory, many scholars have successfully applied reproducing kernel method to solve problems $[12,13,14,15,17,18]$. But the traditional reproducing kernel method [11] is difficult to deal with the integral term, while the HPM can be effectively dealt with the integral term. Because the traditional reproducing kernel method needs orthogonalization, the calculation method is complex and time-consuming. Our method avoids the Smith orthogonalization process in order to save the calculation time and running memory. This article discusses the nonlinear V-FIDE by using SRKM and HPM in the reproducing kernel space, so that the equation can achieve higher accuracy.

In this paper, we describe the homotopy perturbation theory in Section 2. The reproducing kernel theory will be shown in Sections 3 and 4 . The last part presents some numerical examples. In the end, we have the conclusions.

\section{Homotopy perturbation method}

For Equation (1.1), we first have to solve the nonlinear part. The homotopy perturbation method provides a good theoretical basis, we embed a small parameter $p(p \in[0,1])$ by constructing a homotopy map

$$
Y u(x)+p H(u(x))=y(x), \quad u(a)=\alpha,
$$

when $p=0$, the Equation (2.1) is an initial value problem:

$$
Y u(x)=y(x), \quad u(a)=\alpha,
$$

when $p=1$, Equation (2.1) is the original problem (1.1). The parameter $p$ changes from 0 to 1 , then the solution $u(x)$ follows the homotopy path from Equation (2.2) to the original problem Equation (2.1). And the solutions that 
satisfy the homotopy path can be expanded into a power series of $p$ :

$$
u(x, p)=\sum_{n=0}^{\infty} p^{n} u_{n}(x) .
$$

In this way, when $p \rightarrow 1$, the approximate solution of the nonlinear operator equation is obtained

$$
u(x)=\lim _{p \rightarrow 1} u(x, p)=\sum_{n=0}^{\infty} u_{n}(x) .
$$

Take the $k$ derivatives of $F, G$ and set $p=0$, then substitute the type into Equation (2.1):

$$
\sum_{n=0}^{\infty} p^{n} Y u_{n}(x)+p \lambda_{1} \int_{0}^{x} K_{1}(x, t) \sum_{k=0}^{\infty} A_{k} p^{k} d t+p \lambda_{2} \int_{0}^{1} K_{2}(x, t) \sum_{k=0}^{\infty} B_{k} p^{k} d t=y(x),
$$

where

$$
\begin{aligned}
& \sum_{k=0}^{\infty} A_{k} p^{k}=F\left(\sum_{n=0}^{\infty} p^{n} u_{n}(x)\right)=\left.\sum_{k=0}^{\infty} \frac{1}{k !} \frac{d^{k}}{d p^{k}} F\left(\sum_{n=0}^{\infty} p^{n} u_{n}(x)\right)\right|_{p=0}, \\
& \sum_{k=0}^{\infty} B_{k} p^{k}=G\left(\sum_{n=0}^{\infty} p^{n} u_{n}(x)\right)=\left.\sum_{k=0}^{\infty} \frac{1}{k !} \frac{d^{k}}{d p^{k}} G\left(\sum_{n=0}^{\infty} p^{n} u_{n}(x)\right)\right|_{p=0} .
\end{aligned}
$$

Comparing the coefficients of $p^{i}$ on both sides of equation and setting them equal, we can get for $k=0$,

$$
Y u_{0}(x)=y(x), \quad u_{0}(a)=\alpha,
$$

for $p^{k+1}$,

$$
\left\{\begin{array}{l}
Y u_{k}(x)=-\lambda_{1} \int_{0}^{x} K_{1}(x, t) \sum_{k=0}^{\infty} A_{k} d t-\lambda_{2} \int_{0}^{1} K_{2}(x, t) \sum_{k=0}^{\infty} B_{k} d t \\
u_{k}(a)=\alpha
\end{array}\right.
$$

Adding the solution $u_{k}$ of Equations (2.3)-(2.4), we obtain the true solution to nonlinear equations

$$
u_{n}(x)=u_{0}(x)+u_{1}(x)+u_{2}(x)+\ldots .
$$

\section{Reproducing kernel Hilbert space}

We will discuss the Equation (1.1) with support of the reproducing kernel space theory.

Definition 1. ( [7]) Let $H$ be the Hilbert space, and the elements in $H$ are complex-valued functions on $X$. If there is a unique function $K_{s}(t)$ for $\forall s \in X$ that satisfies

$$
\left\langle f, K_{s}\right\rangle=f(s), \quad f \in H .
$$

Then $H$ is defined as reproducing kernel space, $K(s, t)=K_{s}(t)$ is defined as reproducing kernel function. 
To solve Equation (1.1), we need to introduce two reproducing kernel spaces next. The inner product and the norm of reproducing kernel Hilbert space $W_{2}^{2}[a, b]$ and $W_{2}^{1}[a, b]$ are defined as

DEFinition 2. ( [7])

$W_{2}^{2}[a, b]=\left\{u(x) \mid u^{\prime}(x)\right.$ is an absolutely continuous real value function, $\left.u^{\prime \prime}(x) \in L^{2}[a, b]\right\}, \quad\langle u, v\rangle_{W_{2}^{2}}=u(a) v(a)+u^{\prime}(a) v^{\prime}(a)+\int_{a}^{b} u^{\prime \prime} v^{\prime \prime} d x$,

$u, v \in W_{2}^{2}[a, b], \quad\|u\|_{W_{2}^{2}}=\sqrt{\langle u(x), u(x)\rangle_{W_{2}^{2}}}$.

DEFINITION 3. ( [7])

$W_{2}^{1}[a, b]=\{u(x) \mid u(x)$ is an absolutely continuous real value function, $\left.u^{\prime}(x) \in L^{2}[a, b]\right\}, \quad\langle u, v\rangle_{W_{2}^{1}}=u(a) v(a)+\int_{a}^{b} u^{\prime} v^{\prime} d x, \quad u, v \in W_{2}^{1}[a, b]$, $\|u\|_{W_{2}^{1}}=\sqrt{\langle u(x), u(x)\rangle_{W_{2}^{1}}}$.

Theorem 1. ([7]) The reproducing kernel function $R_{s}(t)$ of $W_{2}^{2}[a, b]$ is defined as

$$
R_{s}(t)= \begin{cases}s t+\frac{s t^{2}}{2}-\frac{t^{3}}{6}, & t \leq s, \\ s t+\frac{t s^{2}}{2}-\frac{s^{3}}{6}, & s \leq t\end{cases}
$$

Theorem 2. ( [7]) The reproducing kernel function $r_{s}(t)$ of $W_{2}^{1}[a, b]$ is defined as

$$
r_{s}(t)=\left\{\begin{array}{l}
1-a+s, t \leq s \\
1-a+t, s \leq t
\end{array}\right.
$$

\section{The combination of HPM and SRKM}

We described the HPM for nonlinear equation in Section 2. Equations (2.3) and (2.4) can be considered as follows

$$
\left\{\begin{array}{l}
Y u_{n}(x)=f(x), \\
u_{n}(a)=\alpha, n=0,1,2, \ldots
\end{array}\right.
$$

Now we introduce reproducing kernel method. Firstly, we define a linear operator $L: W_{2}^{2}[a, b] \rightarrow W_{2}^{1}[a, b]$,

$$
L u(x)=Y u_{n}(x), u(x) \in W_{2}^{2}[a, b] .
$$

Therefore, Equation (4.1) can be expressed as

$$
\left\{\begin{array}{l}
L u(x)=f(x) \\
u_{n}(a)=\alpha, n=0,1,2, \ldots
\end{array}\right.
$$


It's easy to prove that $L$ is a bounded linear operator. $L^{*}$ is the adjoint operator of $L$. Let $\phi_{i}(x)=L^{*} r_{s}(t)\left(x_{i}\right), i=1,2, \ldots$, where $L^{*} r_{s}(t)\left(x_{i}\right)=$ $\left\langle L^{*} r_{s}, R_{t}\right\rangle_{W_{2}^{2}}=\left\langle r_{s}, L R_{t}\right\rangle_{W_{2}^{1}}=\left(L R_{t}\right)(s)=\left(L R_{s}\right)(t)$, and $\left\{x_{i}\right\}$ is subset on $[a, b]$.

Theorem 3. $\left\{x_{i}\right\}_{i=1}^{\infty}$ in $[a, b]$ is a set of mutually distinct dense points, then $\left\{\psi_{i}(x)\right\}_{i=1}^{\infty}$ is a complete system on $W_{2}^{2}[a, b]$.

Proof. Assume $\sum_{i=1}^{n} c_{i} \psi_{i}(x)=0$, because $L$ is invertible, and

$$
\sum_{i=1}^{n} c_{i} \psi_{i}(x)=\sum_{i=1}^{n} c_{i} L R_{x_{i}}(x)=L\left(\sum_{i=1}^{n} c_{i} R_{x_{i}}(x)\right)=0 .
$$

In addition, for $u(x) \in W_{2}^{2}[a, b]$, if $\left\langle u(x), \psi_{i}\right\rangle_{W_{2}^{2}}=u\left(x_{i}\right)=0, i=1,2, \ldots$, then $u(x) \equiv 0$.

Let $\psi_{1}(x)=R(x, a)$ and $S_{n+1}=\operatorname{span}\left\{\phi_{1}(x), \phi_{2}(x), \ldots, \phi_{n}(x), \psi_{1}(x)\right\}$. We can obtain the following conclusions:

Theorem 4. Define $P_{n}: W_{2}^{2}[a, b] \rightarrow S_{n+1}[a, b]$, then $u_{n}=P_{n} u$ satisfies:

$$
\left\langle u_{n}, \phi_{k}\right\rangle=f\left(x_{k}, u\left(x_{k}\right)\right), \quad k=1,2, \ldots, n, \quad\left\langle u_{n}, \psi_{1}\right\rangle=\alpha .
$$

Proof.

$$
\begin{aligned}
\left\langle P_{n} u, \phi_{k}\right\rangle_{W_{2}^{2}} & =\left\langle u, \phi_{k}\right\rangle_{W_{2}^{2}}=\left\langle u, L^{*} r_{x_{k}}\right\rangle_{W_{2}^{2}}=\left\langle L u, r_{x_{k}}\right\rangle_{W_{2}^{1}}=L u\left(x_{k}\right) \\
& =f\left(x_{k}, u\left(x_{k}\right)\right), \quad k=1,2, \ldots, n . \\
\left\langle P_{n} u, \psi_{1}\right\rangle_{W_{2}^{2}} & =\left\langle u, P_{n} \psi_{1}\right\rangle_{W_{2}^{2}}=\left\langle u, \psi_{1}\right\rangle_{W_{2}^{2}}=\left\langle u, R_{a}\right\rangle_{W_{2}^{2}}=u(a)=\alpha .
\end{aligned}
$$

Theorem 5. The approximate solution $u_{n}(x)$ uniformly converges to $u(x)$ on $[a, b]$.

Proof. $\left\|u_{n}-u\right\| \rightarrow 0$ holds as $n \rightarrow \infty$ in $W_{2}^{2}[a, b]$. According to the reproducibility of the reproducing kernel function, we have

$$
u_{n}(x)-u(x)=\left\langle u_{n}-u, R_{x}(y)\right\rangle,
$$

thus

$$
\left|u_{n}(x)-u(x)\right|=\left|\left\langle u_{n}-u, R_{x}(y)\right\rangle_{W_{2}^{2}}\right| \leq\left\|u_{n}-u\right\|_{W_{2}^{2}}\left\|R_{x}(y)\right\|_{W_{2}^{2}},
$$

because of the boundedness of the reproducing kernel function, $\left\|R_{x}(y)\right\| \leq M_{0}$, then

$$
\left|u_{n}(x)-u(x)\right| \leq M_{0}\left\|u_{n}-u\right\|_{W_{2}^{2}} \rightarrow 0
$$


Consequently, the exact solution $u_{n} \in S_{n+1}$ of Equation (4.2) can be developed as follows

$$
u_{n}(x)=\sum_{k=1}^{n} q_{k} \phi_{k}(x)+p_{1} \psi_{1} .
$$

Then applying the form Equation (4.4) to Equation (4.3), we can obtain

$$
\begin{aligned}
& p_{1}\left\langle\psi_{1}, \psi_{1}\right\rangle+\sum_{k=1}^{n} q_{j}\left\langle\phi_{k}(x), \psi_{1}\right\rangle=\alpha, \\
& p_{1}\left\langle\psi_{1}, \phi_{j}\right\rangle+\sum_{k=1}^{n} q_{j}\left\langle\phi_{k}(x), \phi_{j}\right\rangle=f\left(x_{j}, u\left(x_{j}\right)\right), \quad j=1,2, \ldots, n .
\end{aligned}
$$

Note that

$$
\begin{aligned}
A & =\left(\begin{array}{ccccc}
\left\langle\psi_{1}, \psi_{1}\right\rangle & \left\langle\phi_{1}, \psi_{1}\right\rangle & \left\langle\phi_{2}, \psi_{1}\right\rangle & \ldots & \left\langle\phi_{n}, \psi_{1}\right\rangle \\
\left\langle\psi_{1}, \phi_{1}\right\rangle & \left\langle\phi_{1}, \phi_{1}\right\rangle & \left\langle\phi_{1}, \phi_{1}\right\rangle & \ldots & \left\langle\phi_{n}, \phi_{1}\right\rangle \\
\left\langle\psi_{1}, \phi_{2}\right\rangle & \left\langle\phi_{1}, \phi_{2}\right\rangle & \left\langle\phi_{2}, \phi_{2}\right\rangle & \ldots & \left\langle\phi_{n}, \phi_{2}\right\rangle \\
\vdots & \vdots & \vdots & \vdots & \vdots \\
\left\langle\psi_{1}, \phi_{n}\right\rangle & \left\langle\phi_{1}, \phi_{n}\right\rangle & \left\langle\phi_{2}, \phi_{n}\right\rangle & \ldots & \left\langle\phi_{n}, \phi_{n}\right\rangle
\end{array}\right), \\
f & =\left(\begin{array}{c}
\alpha \\
f\left(x_{1}, u\left(x_{1}\right)\right) \\
f\left(x_{2}, u\left(x_{2}\right)\right) \\
\vdots \\
f\left(x_{n}, u\left(x_{n}\right)\right)
\end{array}\right) .
\end{aligned}
$$

Thus, we just have to compute $\left(p_{1}, q_{1}, q_{2}, \ldots, q_{n}\right)^{T}=A^{-1} f$.

\section{$5 \quad$ Numerical examples}

The main methods used in this work have been described in the previous sections, some numerical examples are given to illustrate its effectiveness. Meanwhile, the red lines in the figure represent the approximate solutions and the blue dots represent the exact solutions. The absolute errors of the exact, the approximate solutions and CPU time (seconds) are listed in the tables. We also used the following formula to calculate the convergence rate $r$ :

$$
r=\log 2 \frac{\left\|e_{n}\right\|}{\left\|e_{2 n}\right\|} .
$$

Example 1. For the following nonlinear V-FIDE:

$$
\left\{\begin{array}{l}
u^{\prime}(x)+u(x)+\frac{1}{2} \int_{0}^{x} x u^{2}(t) d t-\frac{1}{4} \int_{0}^{1} t u^{3}(t) d t=y(x), \\
u(0)=0
\end{array}\right.
$$

where $y(x)=2 x+x^{2}+\frac{1}{10} x^{6}-\frac{1}{32}$, the exact solution is $u(x)=x^{2}$ (see Figure $1(\mathrm{a}))$. The comparison of the numerical results and the absolute error are listed in Table 1, we get an exact solution with higher precision than the method of hybrid Legendre polynomials and Block-Pulse functions [9] for $n=12$. 


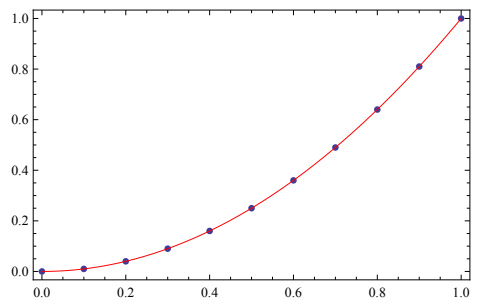

a)

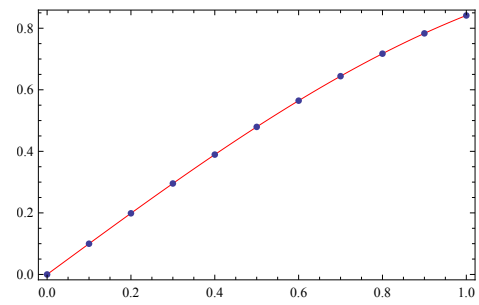

b)

Figure 1. Approximate solutions: a) Example 1, b) Example 2.

Table 1. Numerical result and absolute error for Example 1.

\begin{tabular}{|c|c|c|c|c|c|}
\hline$x$ & Exact solution & Presented method & In $[9]$ & Absolute error in [9] & Absolute errol \\
\hline 0.0 & 0.000000 & 0.000000 & 0.000000 & 0.00000 & 0.00000 \\
\hline 0.1 & 0.010000 & 0.010044 & 0.010917 & $9.100 \mathrm{E}-4$ & $4.4259 \mathrm{E}-5$ \\
\hline 0.2 & 0.040000 & 0.040094 & 0.041703 & $1.703 \mathrm{E}-3$ & $9.3974 \mathrm{E}-5$ \\
\hline 0.3 & 0.090000 & 0.090146 & 0.092364 & $2.364 \mathrm{E}-3$ & $1.4657 \mathrm{E}-4$ \\
\hline 0.4 & 0.160000 & 0.160198 & 0.162911 & $2.911 \mathrm{E}-3$ & $1.9813 \mathrm{E}-4$ \\
\hline 0.5 & 0.250000 & 0.250243 & 0.253371 & $3.371 \mathrm{E}-3$ & $2.4307 \mathrm{E}-4$ \\
\hline 0.6 & 0.360000 & 0.360289 & 0.364244 & $4.244 \mathrm{E}-3$ & $2.8891 \mathrm{E}-4$ \\
\hline 0.7 & 0.490000 & 0.490346 & 0.493830 & $3.830 \mathrm{E}-3$ & $3.4553 \mathrm{E}-4$ \\
\hline 0.8 & 0.640000 & 0.640408 & 0.642375 & $2.375 \mathrm{E}-3$ & $4.0786 \mathrm{E}-4$ \\
\hline 0.9 & 0.810000 & 0.810468 & 0.810337 & $3.370 \mathrm{E}-4$ & $4.6836 \mathrm{E}-4$ \\
\hline 1.0 & 1.000000 & 1.000520 & 0.998506 & $1.494 \mathrm{E}-3$ & $5.1647 \mathrm{E}-4$ \\
\hline$r$ & & $r_{6}=1.99$ & & $r_{12}=1.99$ & \\
\hline $\mathrm{CPU}$ & & & $1.75 \mathrm{~s}$ & & \\
\hline
\end{tabular}

Example 2. For the following V-FIDE:

$$
\left\{\begin{array}{l}
u^{\prime}(x)+u(x)-2 \int_{0}^{x} \sin (x) u^{2}(t) d t=\cos (x)+(1-x) \sin (x)+\cos (x) \sin ^{2}(x), \\
u(0)=0 .
\end{array}\right.
$$

The exact solution is $u(x)=\sin (x)$ (see Fig. 1 (b))

Table 2 illustrates the numerical results and the absolute error. From the Table 2 results, we can see that our method approximates the exact solution more closely than the hybrid Legendre polynomials and Block-Pulse functions [9] for $n=12$.

Example 3. Consider the nonlinear V-FIDE:

$$
\left\{\begin{array}{l}
u^{\prime}(x)+\int_{0}^{x}\left(u^{2}(t)-2\right) d t=\frac{1}{5} x^{5} \\
u(0)=0
\end{array}\right.
$$

with the exact solution given by $u(x)=x^{2}$. The comparison of the numerical results and the absolute error are listed in Table 3, our method is more accurate than the method of Laplace discrete adomian decomposition in [4] for $n=4$. 
Table 2. Numerical result and absolute error for Example 2.

\begin{tabular}{cccccc}
\hline$x$ & Exact solution & Presented method & In [9] & Absolute error in [9] & Absolute error \\
\hline 0.0 & 0.000000 & 0.000000 & 0.000032 & $3.2 \mathrm{E}-5$ & $1.82077 \mathrm{E}-14$ \\
0.1 & 0.099833 & 0.099793 & 0.099435 & $3.98417 \mathrm{E}-4$ & $3.99348 \mathrm{E}-5$ \\
0.2 & 0.198669 & 0.198584 & 0.198304 & $3.65331 \mathrm{E}-4$ & $8.53956 \mathrm{E}-5$ \\
0.3 & 0.295520 & 0.295387 & 0.295493 & $2.72067 \mathrm{E}-4$ & $1.33170 \mathrm{E}-4$ \\
0.4 & 0.389418 & 0.389239 & 0.389688 & $2.69658 \mathrm{E}-4$ & $1.79180 \mathrm{E}-4$ \\
0.5 & 0.479425 & 0.479207 & 0.479311 & $1.14539 \mathrm{E}-4$ & $2.18684 \mathrm{E}-4$ \\
0.6 & 0.564642 & 0.564383 & 0.562965 & $1.67747 \mathrm{E}-3$ & $2.59684 \mathrm{E}-4$ \\
0.7 & 0.644217 & 0.643906 & 0.640005 & $4.21269 \mathrm{E}-3$ & $3.11780 \mathrm{E}-4$ \\
0.8 & 0.717356 & 0.716984 & 0.708103 & $9.25309 \mathrm{E}-3$ & $3.71839 \mathrm{E}-4$ \\
0.9 & 0.783326 & 0.782891 & 0.764843 & $1.84839 \mathrm{E}-2$ & $4.36266 \mathrm{E}-4$ \\
1.0 & 0.841470 & 0.840970 & 0.807845 & $3.36260 \mathrm{E}-2$ & $5.01073 \mathrm{E}-4$ \\
\hline$r$ & $r_{6}=2.00$ & & $r_{12}=1.99$ & \\
\hline $\mathrm{CPU}$ & \multicolumn{5}{c}{$0.923 s$} \\
\hline
\end{tabular}

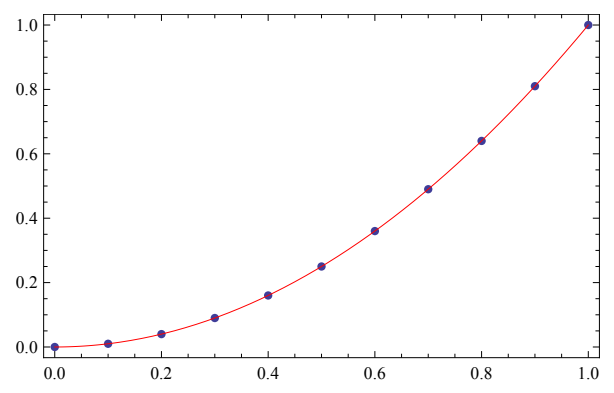

Figure 2. Approximate solution of Example 3.

Table 3. Numerical result and absolute error for Example 3.

\begin{tabular}{cccccc}
\hline$x$ & Exact sol. & Presented method & In [4] & Abs. error in $[4]$ & Abs. error \\
\hline 0.0 & 0.00000000 & 0.00000000 & 0.00000000 & 0.00000000 & 0.00000000 \\
0.2 & 0.04000000 & 0.040000000 & 0.03999986 & $1.41640 \mathrm{E}-7$ & $4.68375 \mathrm{E}-17$ \\
0.4 & 0.16000000 & 0.160000000 & 0.15999094 & $9.05930 \mathrm{E}-6$ & $2.77556 \mathrm{E}-17$ \\
0.6 & 0.36000000 & 0.360000000 & 0.35989712 & $1.02879 \mathrm{E}-4$ & $1.11022 \mathrm{E}-16$ \\
0.8 & 0.64000000 & 0.64000000 & 0.63942742 & $5.72582 \mathrm{E}-4$ & $2.22045 \mathrm{E}-16$ \\
1.0 & 1.00000000 & 1.00000000 & 0.99787295 & $2.12705 \mathrm{E}-3$ & $1.11022 \mathrm{E}-15$ \\
\hline $\mathrm{CPU}$ & \multicolumn{5}{c}{$0.579 s$} \\
\hline
\end{tabular}

\section{Conclusions}

In this article, the SRKM and the HPM were successfully applied to figure out the nonlinear V-FIDE by getting the uniform approximate solution. Besides, compared with the method of Hybrid Legendre polynomials [9], Laplace discrete adomian decomposition method [4], the convergence speed and accuracy of solution were better. 


\section{Acknowledgements}

Correspondence to Jing Niu, School of Mathematics and Sciences, Harbin Normal University, Harbin, 150025, China. This research were supported by the Heilongjiang Province with College Innovative Talent foundation of China(Grant Number UNPYSCT-2017179).

\section{References}

[1] A. Abdi and S.A. Hosseini. The barycentric rational differencequadrature scheme for systems of Volterra integro-differential equations. SIAM Journal on Scientific Computing, 40(3):A1936-A1960, 2018. https://doi.org/10.1137/17M114371X.

[2] E. Babolian, Z. Masouri and S. Hatamzadeh-Varmazyar. New direct method to solve nonlinear Volterra-Fredholm integral and integro differential equation using operational matrix with block-pulse functions. Progress in Electromagnetics Research B, 8:59-76, 2008. https://doi.org/10.2528/pierb08050505.

[3] E. Babolian, Z. Masouri and S. Hatamzadeh-Varmazyar. Numerical solution of nonlinear Volterra-Fredholm integro-differential equations via direct method using triangular functions. Computers Mathematics With Applications, 58(2):239247, 2009. https://doi.org/10.1016/j.camwa.2009.03.087.

[4] H.O. Bakodah, M. Almazmumy, S.O. Almuhalbedi and L. Abdullah. Laplace discrete Adomian decomposition method for solving nonlinear integro differential equations. Journal of Applied Mathematics and Physics, 7(6):1388-1407, 2019. https://doi.org/10.4236/jamp.2019.76093.

[5] J. Biazar, B. Ghanbari, M.G. Porshokouhi and M.G. Porshokouhi. Hes homotopy perturbation method: A strongly promising method for solving non-linear systems of the mixed VolterraFredholm integral equations. Computers Mathematics with Applications, 61(4):1016-1023, 2011. https://doi.org/10.1016/j.camwa.2010.12.051.

[6] N. Bildik. Modified decomposition method for nonlinear VolterraFredholm integral equations. Chaos Solitons Fractals, 33:308-313, 2007. https://doi.org/10.1016/j.chaos.2005.12.058.

[7] M.G. Cui and Y.Z. Lin. Nonlinear Numerical Analysis in the Reproducing Kernel Space. Nova Science Publishers, New York, London, 2009.

[8] M. Ghasemi, M.T. Kajani and E. Babolian. Application of Hes homotopy perturbation method to nonlinear integro-differential equations. Applied Mathematics and Computation, 188(1):538-548, 2007. https://doi.org/10.1016/j.amc.2006.10.016.

[9] K. Maleknejad, B. Basirat and E. Hashemizadeh. Hybrid legendre polynomials and block-pulse functions approach for nonlinear V0olterra-Fredholm integrodifferential equations. Computers Mathematics with Applications, 61(9):28212828, 2011. https://doi.org/10.1016/j.camwa.2011.03.055.

[10] K. Maleknejad and E. Saeedipoor. Convergence analysis of hybrid functions method for two-dimensional nonlinear VolterraFredholm integral equations. Journal of Computational and Applied Mathematics, 368:1-10, 2020. https://doi.org/10.1016/j.cam.2019.112533. 
[11] J. Niu, Y.Z. Lin and C.P. Zhang. Approximate solution of nonlinear multi-point boundary value problem on the half-line. Mathematical Modelling and Analysis, 17(2):190-202, 2012. https://doi.org/10.3846/13926292.2012.660889.

[12] J. Niu, L.X. Sun, M.Q. Xu and J.J. Hou. A reproducing kernel method for solving heat conduction equations with delay. Applied Mathematics Letters, 100:106036, 2019. https://doi.org/10.1016/j.aml.2019.106036.

[13] J. Niu, M.Q. Xu and G.M. Yao. An efficient reproducing kernel method for solving the AllenCahn equation. Applied mathematics letters, 89:78-84, 2019. https://doi.org/10.1016/j.aml.2018.09.013.

[14] K. Ozen and K. Orucoglu. Approximate solution to a multi-point boundary value problem involving nonlocal integral conditions by reproducing kernel method. Mathematical Modelling and Analysis, 18(4):529-536, 2013. https://doi.org/10.3846/13926292.2013.840867.

[15] M.Q. Xu, L.F. Zhang and E. Tohidi. A fourth-order least-squares based reproducing kernel method for one-dimensional elliptic interface problems. Applied Numerical Mathematics, 162:124-136, 2021. https://doi.org/10.1016/j.apnum.2020.12.015.

[16] S.A. Yousefi and M. Razzaghi. Legendre wavelets method for the nonlinear Volterra-Fredholm integral equations. Mathematics and Computers in Simulation, 70(1):1-8, 2005. https://doi.org/10.1016/j.matcom.2005.02.035.

[17] Z.H. Zhao, Y.Z. Lin and J. Niu. Convergence order of the reproducing kernel method for solving boundary value problems. Mathematical Modelling and Analysis, 21(4):466-477, 2016. https://doi.org/10.3846/13926292.2016.1183240.

[18] H. Zhu, J. Niu, R.M. Zhang and Y.Z. Lin. A new approach for solving nonlinear singular boundary value problems. Mathematical Modelling and Analysis, 23(1):33-43, 2018. https://doi.org/10.3846/mma.2018.003. 